\title{
Multifractal Detrended Cross-correlation Analysis of Gold and WTI Crude Oil Price Time Series
}

\section{Burugupalli $\mathbf{S}^{*}$}

Center for Integrated Studies, University of Hyderabad, Gachibowli, Hyderabad-500 046, India

\begin{abstract}
Several papers have documented the cross-correlations across wide range of markets using different methods and the results are being used for the improvisation of market efficiency. In this paper, we have investigated the crosscorrelations between Gold market and WTI Crude oil market using MF-X-DFA. Quantitatively, we have employed the recently developed MF-X-DFA method for the data of Gold and WTI Crude oil markets and we have confirmed the existence of cross-correlation between Gold and Crude oil markets. We have also found that these cross-correlations are strongly multifractal in the short term and weakly multifractal in long the term. Moreover, their behavior for small fluctuations is persistent and those of large fluctuations are anti persistent in the short term. Cross-correlations of very small fluctuations and large fluctuations are persistent but whereas for small fluctuations are anti-persistent in the long term. Furthermore, based on multifractal spectrum, we have produced substantial evidences to determine crosscorrelation behaviors exhibit multifractal features. Our results have significant implications to market efficiency.
\end{abstract}

Keywords: Non-stationary time series; Fractals; Hurst exponent; Multifractal detrended Cross-correlation Analysis

\section{Introduction}

The multifractality in real world non stationary time series data are described better from scaling exponents. The observed signals of the physical quantities characterizing any complex system like social, financial, ecological, biological or technological are composed of large number of interacting assorted parameters linked each other nonlinearly and they exhibit long-range correlations. It is of crucial importance and significance to quantify such long-range correlations to have a deep understanding of the dynamics of the underlying complex systems. The theory of fractals proposed by Mandelbrot [1] in contrast to the efficient market hypothesis leads to study of complex system behavior through different method development and approaches. Hurst $[2,3]$ proposed rescaled range analysis $(\mathrm{R} / \mathrm{S})$, the most popular analysis method for fractals. To overcome R/S analysis method sensitiveness to short-term auto-correlation and nonstationary nature causing bias error; detrended fluctuation analysis (DFA) method was proposed [4] which applied long-range power-law correlation. DFA and its multifractal generalization MF-DFA methods [5] are widely used to describe the fractal properties. Many researchers studied multifractal properties on the different non stationary natural and financial time series data like biological data [6], exchange rate market [7], stock market $[8,9]$ gold market $[8,10]$, crude oil market [9-12]. Two major sources of multifractality which can be found in various time series: one is nonlinear temporal correlation for small and large fluctuations; while the other is fat-tailed probability distribution of increments $[8,13,14]$.

Also multifractality in the daily returns of emerging European stock markets is studied using the Empirical Mode Decomposition (EMD) based Multifractal Detrended Fluctuation Analysis [15]. A comparison study of different fluctuation methods for analyzing nonstationary time series were studied for efficacy of the methods like Fluctuation Analysis (FA); Detrended Fluctuation Analysis (DFA) and Detrended Moving Average (DMA). It was reported that [16] Centred Detrending Moving Average (CDMA) and DFA are the Methods of Choice in determining the Hurst index of time series. CDMA has the best performance while DFA is only slightly worse in some situations.
Also CDMA and DFA are less sensitive to the scaling range compared to other methods like FA.

Cross-correlation found to be exists in many simultaneously recorded time series in various real world commodities or financial market data. Therefore, in recent years, many researchers attempted to quantify cross-correlations between non stationary data from complex systems. Detrended Cross-Correlation Analysis (DCCA) was proposed [17] to investigate power-law cross-correlations between two simultaneously recorded time series in the presence of non-stationarity. This new method was applied to different time series for longrange power-law cross-correlations [18]. Subsequently, multifractal generalization of the method was proposed [19] describing the new method as multifractal detrended cross-correlation analysis (MFDCCA) by combining MFDFA and DCCA approaches. The MFDCCA method was applied [13,20-26] to many different time series for cross correlation analysis.

Efficiency of Brent and WTI crude oil market was analyzed by means of R/S analysis [27]. Dynamics of crude oil prices was studied thru stochastic multi-model approach [28]. Market agent perspective on fractal features in crude oil time series was studied $[29,30]$. From the analysis it was evidence that the crude oil market is a persistent process with long-range memory effects with multifractal structures. The MFDFA analysis on gold price revealed that multifractality is mainly due to the temporal correlation [30]. But there exist a cross correlation between the gold and Crude oil time series to study market behavior

*Corresponding author: Burugupalli S, Assistant Professor, Center for Integrated Studies, University of Hyderabad, Gachibowli, Hyderabad-500 046 India, Tel: 91-9581042443; E-mail: satish.burugupalem@gmail.com

Received September 10, 2014; Accepted December 30, 2014; Published January 15, 2015

Citation: Burugupalli S(2015) Multifractal Detrended Cross-correlation Analysis of Gold and WTI Crude Oil Price Time Series. J Bus Fin Aff 3: 130. doi:10.4172/21670234.1000130

Copyright: ( 2015 Burugupalli S. This is an open-access article distributed under the terms of the Creative Commons Attribution License, which permits unrestricted use, distribution, and reproduction in any medium, provided the original author and source are credited. 
and underlying complexity. This article is focused on that study to analyze the cross correlation between Gold and Crude Oil. The section 2 of the article presents the MFD CCA procedure while the Section 3 describes the data. The Section 4 shares the result and its analysis. Section 5 gives our conclusions.

\section{Methodology}

Assume that there are two series $x(i)$ and $y(i)$ where $i=1,2, \ldots, N$. Now the MFD CCA method is as follows.

Step 1. Construct the profile

$$
\begin{aligned}
(i) & =\sum(x(t)-\bar{x}) \\
\mathrm{Y}(i) & =\sum(y(t)-\bar{y})
\end{aligned}
$$

Where $\bar{x}$ and $\bar{y}$ are the average of the two time series $\mathrm{x}(\mathrm{i})$ and $\mathrm{y}(\mathrm{i})$.

Step 2. The profiles $\mathrm{X}(\mathrm{i})$ and $\mathrm{Y}(\mathrm{i})$ are divided into $\mathrm{N}_{\mathrm{s}}=[\mathrm{N} / \mathrm{s}]$ nonoverlapping windows of equal length s. Since the length $\mathrm{N}$ is not always a multiple of the considered time scale s hence in order not to discard the section of series, the same procedure is repeated starting from the reverse end of each profile. Thus, $2 \mathrm{~N}_{\mathrm{s}}$ non-overlapping windows are obtained together.

Step 3. The local trends $\mathrm{X} v$ (i) and $\mathrm{Y} v$ (i) for each segment $v$ (where, $v=1,2,3, \ldots, 2 \mathrm{~N}_{\mathrm{s}}$ ) are evaluated by least squares fits of the data, then the detrended covariance is determined by

$$
F^{2}(s, v)=\frac{1}{s} \sum_{i=1}^{t}\left|X((v-1) s+i)-X^{v}(i)\right| \square\left|Y((v-1) s+i)-Y^{v}(i)\right|
$$

Here for each segment $\mathrm{v}, \mathrm{v}=1,2, \ldots \mathrm{N}_{\mathrm{s}}$ hence,

$$
F^{2}(s, v)=\frac{1}{s} \sum_{i=1}^{t}\left|X\left(N-\left(v-N_{s}\right) s+i\right)-X^{v}(i)\right| \square\left|Y\left(N-\left(v-N_{s}\right) s+i\right)-Y^{v}(i)\right|
$$

Here for each segment $\mathrm{v}, \mathrm{v}=\mathrm{N}_{\mathrm{s}}+1, \mathrm{~N}_{\mathrm{s}}+2, \ldots, 2 \mathrm{~N}_{\mathrm{s}}$. The trends $\mathrm{X}^{\mathrm{v}}(\mathrm{i})$ and $\mathrm{Y}^{\mathrm{v}}(\mathrm{i})$ are the fitting polynomial with order $\mathrm{m}$ in each segment $\mathrm{v}$.

Step 4 . Hence the $\mathrm{q}^{\text {th }}$ order fluctuation function would be,

$$
F_{q}(s)=\left[\frac{1}{2 N_{s}} \sum_{v=1}^{2 N_{s}}\left[F^{2}(s, v)\right]^{q / 2}\right]^{1 / q}
$$

If $q \neq 0$ then the $\mathrm{q}^{\text {th }}$ order fluctuation function would be,

$$
F_{0}(s)=\exp \left\{\frac{1}{4 N_{s}} \sum_{v=1}^{2 N_{s}} \ln \left[F^{2}(s, v)\right]\right\}
$$

\section{When $\mathrm{q}=2$ the MFD CCA is as like CCA}

Step 5. Analyze the scaling behavior of the fluctuations by observing logarithmic plots between $\mathrm{F}_{\mathrm{q}}(\mathrm{s})$ and $\mathrm{s}$ for each values of $\mathrm{q}$. If the two series are long-range cross-correlated then the $\mathrm{F}_{\mathrm{q}}(\mathrm{s})$ will increase for values of $\mathrm{s}$ and we can obtain a power-law expression as

$$
F_{q}(s) \sim s^{H_{x y}(q)}
$$

Hence taking log on both sides, we could represent this as

$$
\log \mathrm{F}_{\mathrm{q}}(\mathrm{s})=\mathrm{H}_{\mathrm{xy}}(\mathrm{q}) \log (\mathrm{s})+\log \mathrm{A}(8)
$$

Here the scaling exponent $\mathrm{H}_{\mathrm{xy}}(\mathrm{q})$ is known as the generalized crosscorrelation Hurst exponent, describing the power-low relationship.
Especially, if the two time series are equal then MFD CCA is the same as MFDFA. When the scaling exponent $\mathrm{H}_{\mathrm{xy}}(\mathrm{q})$ is independent $\mathrm{q}$, the cross-correlation between two series is monofractal while if the scaling exponent $\mathrm{H}_{\mathrm{xy}}(\mathrm{q})$ is dependent on $\mathrm{q}$, then the cross-correlation between two series is multifractal. If the scaling exponent $\mathrm{H}_{\mathrm{xy}}(\mathrm{q}=2)>0.5$ then the cross-correlations between the return fluctuation from the two series related to $\mathrm{q}$ are long-range persistent. If the scaling exponent $\mathrm{H}_{\mathrm{xy}}(\mathrm{q}=2)<0.5$, the cross-correlations between the return fluctuations of the two series related to $\mathrm{q}$ are anti-persistent. If $\mathrm{H}_{\mathrm{xy}}(\mathrm{q}=2)=0.5$, there are no cross-correlations between the two series. Further for positive $\mathrm{q}$, the $\mathrm{H}_{\mathrm{xy}}(\mathrm{q})$ describes the scaling behavior of the segments with large fluctuations. On the contrary, for negative $q, \mathrm{H}_{x y}(q)$ describes the scaling behavior of the segments with small fluctuations. According to the multifractal formalism, the Renyi exponent $\tau_{x y}(q)$ can be used to characterize the multifractal nature of the time series which is expressed as,

$$
\tau_{x y}(q)=q H_{x y}(q)-1
$$

If the Renyi exponent $\tau_{x y}(q)$ is linear of the q, we can conclude that the two correlated series is monofractal, otherwise, it is multifractal The multifractal spectra $\mathrm{f}_{\mathrm{xy}}(\alpha)$ describes the singularity content of the time series which could be derived through the Legendre transform:

$$
\begin{aligned}
& \alpha_{x y}(q)=H_{x y}(q)+q H_{x y}^{\prime}(q) \\
& f_{x y}(\alpha)=q\left(\alpha_{x y}-H_{x y}(q)\right)+1
\end{aligned}
$$

\section{Data Analysis}

Although crude oil prices possess long-range dependence, the degree of long-range has decreased in short time horizons, although the market is tending towards efficiency regime at long. Crude oil is called the blood of industries which plays an important role in all economies driving backbone of financial system. Oil price, as one of the main focal points in many countries, has become an increasingly essential topic of concern to governments, enterprises and investors. Therefore, understanding the dynamics of its price time series seems to be crucial, since it may allow one to assess the potential impacts of its shocks on several economies and on other financial assets. Similarly Gold price fluctuation and trading is directly related to market inflation in any country. So it becomes evident for a cross correlation study and analysis on Gold and Crude Oil time series.

Global crude oil pricing benchmarks Brent and WTI price hence its price volatility become a hot research topic recently. The US's imported crude oil is priced in accordance with WTI price, so the change of WTI crude oil price reflects the US's crude oil demand. Crude oil outside the US market is priced in accordance with Brent price, so Brent price can reflect international crude oil demand more deeply than WTI. For two markets, maybe Brent market is affected more by Gulf Wars than WTI hence to avoid impact of gulf war onto Crude oil price we had chosen WTI Crude oil time series for cross correlation study with Gold time series.

We choose the daily closing data of West Texas Intermediate (WTI) crude oil obtained from the Energy Information Administration, US Department of Energy (http://www.eia.gov/dnav/pet/pet_pri_spt_ s1_d.htm) and the Gold data obtained from (http://fx.sauder.ubc. ca/data.html) for the period of 3rd January 1995 to $13^{\text {th }}$ August 2013 with total 4486 data points. The return of daily price is calculated as, $r(t)=\log \left(P_{t}\right)-\log \left(\left(P_{t-1}\right)\right.$ where $P_{t}$ being the daily closing price index at time $t$ (Figure 1). 
Citation: Burugupalli S (2015) Multifractal Detrended Cross-correlation Analysis of Gold and WTI Crude Oil Price Time Series. J Bus Fin Aff 3: 130. doi:10.4172/2167-0234.1000130

Page 3 of 5
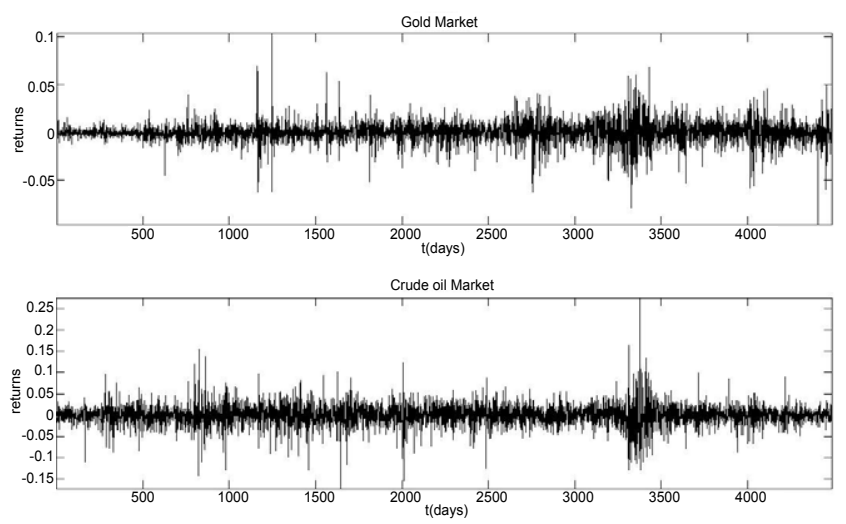

Figure 1: Returns of Gold and WTI Crude oil markets.

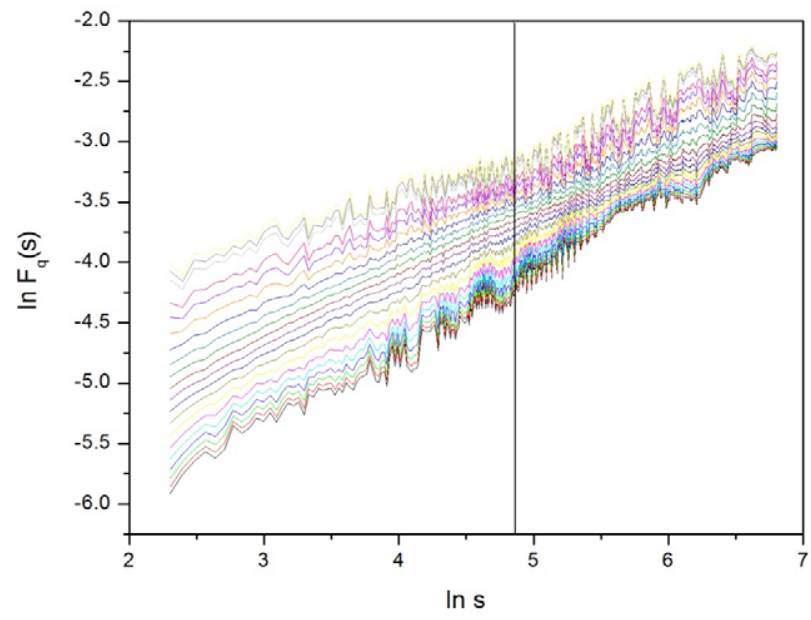

Figure 2: Fluctuation function.

\section{Results and Discussion}

To test the presence of cross-correlation quantitatively we need MF-X-FA method which can estimate the cross-correlation exponent. We show the log-log plots of fluctuation function $F_{q}(s)$ versus time scale $s$ for Gold and WTI Crude oil markets. In Figure 2 we can find that only one line cannot fit the log-log plots of $\mathrm{F}_{\mathrm{q}}(\mathrm{s})$ versus time scale $s$ well. Hence we define the "crossover" $s$, as the turning point when the linear trend of the curves underwent a fundamental change. The short-term behavior of financial market is easily influenced by the market external factors such as the major events while the long-term behavior is determined by the internal factors. With the time evolving, the short-term shocks gradually decay for the effects of long-term supply and demand mechanism in the markets. The scaling exponents for $s<s^{\star}$ can reflect the short range correlation also imply the correlated behaviors in the short-term. Then, the scaling exponents for $\mathrm{s}>\mathrm{s}^{*}$ imply the correlated behaviors in the long-term (Table 1). Thus, we can say that the "crossover" can reflect the lasting period of the effects of the factors which determine the market short-term behavior. We found the "crossover" at about $\ln \left(\mathrm{s}^{\star}\right)=4.8$ (i.e. 130 days). We provide the slopes of each line, just the scaling exponents for $\mathrm{s}<\mathrm{s}^{\star}$ and $\mathrm{s}>\mathrm{s}^{\star}$ in the Tables 2 and 3 respectively.

For $\mathrm{q}=2$, the scaling exponent is the Hurst exponent calculated

\begin{tabular}{|l|c|c|c|c|c|c|}
\hline & Mean & Min & Max & Stand dev & Skewness & Kurtosis \\
\hline Gold & $2.78 \mathrm{E}-04$ & -0.0960 & 0.1031 & 0.0113 & -0.1481 & 10.665 \\
\hline Crude oil & $4.04 \mathrm{E}-04$ & -0.1709 & 0.2756 & 0.0256 & 0.0673 & 10.4602 \\
\hline
\end{tabular}

Note:*min, max, stand dev denote minimum, maximum, standard deviation respectively.

Table 1: Descriptive statistics for the returns of Gold and WTI Crude oil markets.

\begin{tabular}{|c|c|c|c|c|}
\hline & $\mathbf{H x x}$ & $\mathbf{H x y}$ & Hyy & {$[\mathbf{H x x + H y y ] / 2}$} \\
\hline-10 & 0.53304 & 0.59079 & 0.75124 & 0.64214 \\
\hline-9 & 0.53012 & 0.58538 & 0.73839 & 0.63425 \\
\hline-8 & 0.52744 & 0.57946 & 0.72295 & 0.6252 \\
\hline-7 & 0.52538 & 0.57318 & 0.70445 & 0.61491 \\
\hline-6 & 0.52455 & 0.56682 & 0.68255 & 0.60355 \\
\hline-5 & 0.52596 & 0.56077 & 0.65727 & 0.59161 \\
\hline-4 & 0.53088 & 0.55523 & 0.62929 & 0.58008 \\
\hline-3 & 0.53938 & 0.54979 & 0.60003 & 0.5697 \\
\hline-2 & 0.54723 & 0.5435 & 0.5711 & 0.55917 \\
\hline-1 & 0.54767 & 0.53551 & 0.54309 & 0.54538 \\
\hline 0 & 0.54064 & 0.5253 & 0.51443 & 0.52754 \\
\hline 1 & 0.53083 & 0.5119 & 0.4809 & 0.50587 \\
\hline 2 & 0.51905 & 0.49334 & 0.43573 & 0.47739 \\
\hline 3 & 0.50321 & 0.46782 & 0.37323 & 0.43822 \\
\hline 4 & 0.48355 & 0.43678 & 0.29869 & 0.39112 \\
\hline 5 & 0.46283 & 0.40544 & 0.22774 & 0.34529 \\
\hline 6 & 0.44344 & 0.37852 & 0.17026 & 0.30685 \\
\hline 7 & 0.42639 & 0.35743 & 0.12695 & 0.27667 \\
\hline 8 & 0.41176 & 0.34142 & 0.09488 & 0.25332 \\
\hline 9 & 0.39929 & 0.32921 & 0.07097 & 0.23513 \\
\hline 10 & 0.38863 & 0.31968 & 0.05279 & 0.22071 \\
\hline & & & & \\
\hline
\end{tabular}

Table 2: Short term H(q) vs q for Gold and WTI Crude oil markets.

\begin{tabular}{|c|c|c|c|c|}
\hline $\mathbf{Q}$ & Hxx & Hxy & Hyy & {$[$ Hxx+Hyy]/2 } \\
\hline-10 & 0.63574 & 0.55373 & 0.497 & 0.56637 \\
\hline-9 & 0.6284 & 0.5448 & 0.49182 & 0.56011 \\
\hline-8 & 0.61945 & 0.53428 & 0.48663 & 0.55304 \\
\hline-7 & 0.60832 & 0.52205 & 0.48167 & 0.54499 \\
\hline-6 & 0.59415 & 0.50826 & 0.47732 & 0.53573 \\
\hline-5 & 0.5758 & 0.49353 & 0.47414 & 0.52497 \\
\hline-4 & 0.55202 & 0.47918 & 0.47301 & 0.51252 \\
\hline-3 & 0.52238 & 0.46722 & 0.47534 & 0.49886 \\
\hline-2 & 0.48898 & 0.46001 & 0.48333 & 0.48616 \\
\hline-1 & 0.4564 & 0.45973 & 0.50016 & 0.47828 \\
\hline 0 & 0.42773 & 0.46786 & 0.52902 & 0.47837 \\
\hline 1 & 0.40273 & 0.48374 & 0.56865 & 0.48569 \\
\hline 2 & 0.38038 & 0.50259 & 0.60833 & 0.49435 \\
\hline 3 & 0.36056 & 0.51691 & 0.63479 & 0.49768 \\
\hline 4 & 0.34357 & 0.52214 & 0.64496 & 0.49427 \\
\hline 5 & 0.3294 & 0.51916 & 0.64383 & 0.48661 \\
\hline 6 & 0.31769 & 0.51136 & 0.6369 & 0.47729 \\
\hline 7 & 0.30797 & 0.50168 & 0.62774 & 0.46785 \\
\hline 8 & 0.29982 & 0.49186 & 0.61818 & 0.459 \\
\hline 9 & 0.29289 & 0.48267 & 0.60908 & 0.45098 \\
\hline 10 & 0.28691 & 0.4744 & 0.60076 & 0.44383 \\
\hline
\end{tabular}

Table 3: Long term H(q) vs q for Gold and WTI Crude oil markets.

from the method of DFA. When $\mathrm{q}=2$, the scaling exponent for Gold and WTI Crude oil markets is markets is 0.49334 for $s<s^{*}$ indicating that Gold and WTI Crude oil markets are weakly cross-correlated in the short-term. For $\mathrm{s}>\mathrm{s}^{*}$, the scaling exponent for Gold and WTI Crude oil markets is 0.50259 , larger than that for $\mathrm{s}<\mathrm{s}^{*}$ indicating that they are moderately cross-correlated in the long-term. 


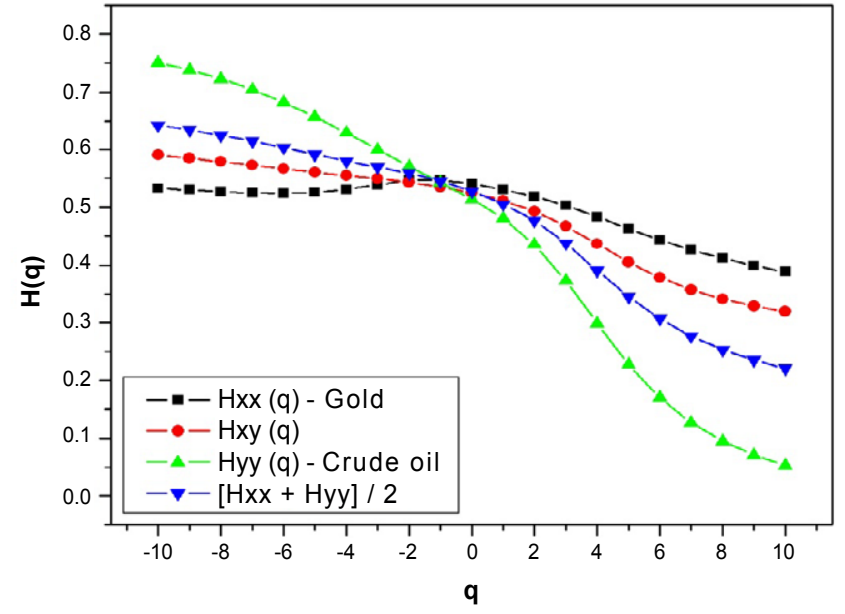

Figure 3: Short term $s<s^{*}$.

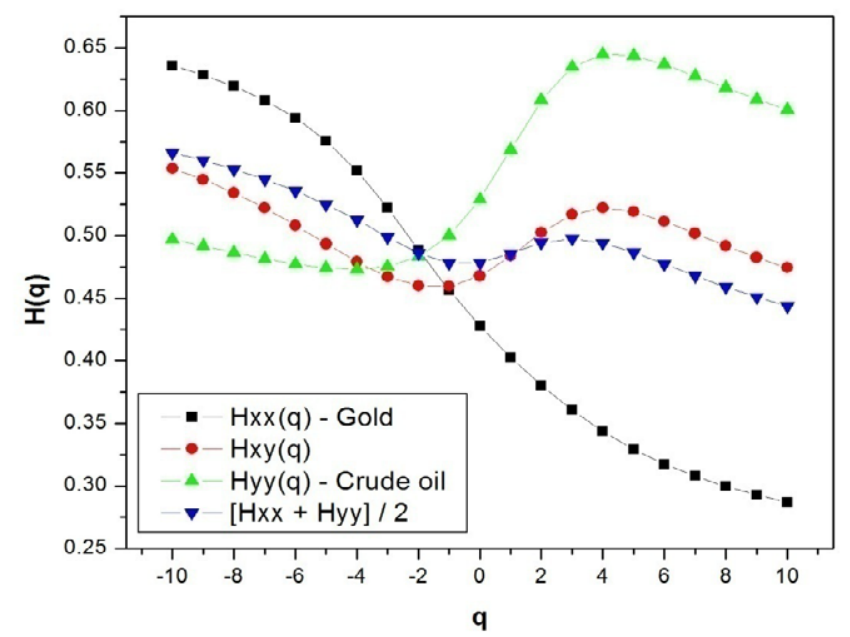

Figure 4: Long term $s<s^{*}$.

For other values of $\mathrm{q}$, the scaling exponents indicate the crosscorrelated behaviors between the kinds of fluctuations related to $\mathrm{q}$ showing multifractlity behavior. We provide the scaling exponents with q varying from -10 to 10 for $\mathrm{H}_{\mathrm{xx}}(\mathrm{q}), \mathrm{H}_{\mathrm{xy}}(\mathrm{q}), \mathrm{H}_{\mathrm{yy}}(\mathrm{q})$, and the average scaling exponents $\left[\mathrm{H}_{x x}(\mathrm{q})+\mathrm{H}_{\mathrm{yy}}(\mathrm{q})\right] / 2$ for short term and long term in Figures 3 and 4 respectively.

From Figure 3 for $s<s^{\star}$, we can find that $H_{x x}(q)$ - Gold market scaling exponents are decreasing from 0.53 to 0.38 indicating that it is exhibiting moderate multifractal features in short term where as $\mathrm{H}_{\text {yy }}$ (q)-WTI Crude oil market scaling exponents decrease from 0.75 to 0.05 indicating it is exhibiting strong multifractal features in short term. While the cross-correlated scaling exponents $\mathrm{H}_{\mathrm{xy}}(\mathrm{q})$ decreases from 0.6 to 0.35 indicating that cross-correlated behaviors are multifractal in short term. Moreover for $\mathrm{q}<0$ we can find that for $\mathrm{H}_{\mathrm{xy}}(\mathrm{q})$, the scaling exponents are greater than 0.5 indicating that crosscorrelated behaviors of small fluctuation are persistent (positive) in the short term. The scaling exponents for $\mathrm{q}>2$ are less than 0.5 indicating that cross-correlated behaviors of large fluctuations are anti-persistent (negative) in the short term.
From Figure 4 for $s>s^{\star}$, we can find that $H_{x x}$ (q)-Gold market scaling exponents are decreasing from over 0.63 to 0.28 indicating it is exhibiting high multifractal features in the long term. Similarly $\mathrm{H}_{\mathrm{yy}}$ (q)-WTI Crude oil market scaling exponents increase from 0.5 to 0.6 indicating it is moderately multifractal. While the cross-correlated scaling exponents $\mathrm{H}_{\mathrm{xy}}(\mathrm{q})$ are not varying considerably indicating crosscorrelated behavior is weakly multifractal in the long term. Moreover for $\mathrm{q}<-5$ the scaling exponents are greater than 0.5 indicating that crosscorrelated behaviors of small fluctuations are persistent (positive) in the long term. Congruently for $\mathrm{q}>1$ the scaling exponents are greater than 0.5 indicating that cross-correlated behaviors of large fluctuations are also persistent (positive) in the long term.

For $\mathrm{q}<0$, the scaling exponents $\mathrm{H}_{\mathrm{xy}}(\mathrm{q})$ are always less than the average scaling exponents $\left[\mathrm{H}_{\mathrm{xx}}(\mathrm{q})+\mathrm{H}_{\mathrm{yy}}(\mathrm{q})\right] / 2$ and for $\mathrm{q}>0$, the scaling exponents $\mathrm{H}_{\mathrm{xy}}(\mathrm{q})$ are greater than the average scaling exponents $\left[\mathrm{H}_{\mathrm{xx}}(\mathrm{q})+\mathrm{H}_{\mathrm{yy}}(\mathrm{q})\right] / 2$ in the short term.

For $\mathrm{q}<1$, the scaling exponents $\mathrm{H}_{\mathrm{xy}}(\mathrm{q})$ are always less than the average scaling exponents $\left[\mathrm{H}_{\mathrm{xx}}(\mathrm{q})+\mathrm{H}_{\mathrm{yy}}(\mathrm{q})\right] / 2$ and for $\mathrm{q}>1$, the scaling exponents $\mathrm{H}_{\mathrm{xy}}(\mathrm{q})$ are greater than the average scaling exponents $\left[\mathrm{H}_{\mathrm{xx}}(\mathrm{q})+\mathrm{H}_{\mathrm{yy}}(\mathrm{q})\right] / 2$ in the long term.

\section{Conclusion}

Historically gold retains its value during times of crisis and is used as a hedge against inflation, deflation or currency devaluation. Gold also most popularly used as an investment. Crude Oil is a vital source of energy for the world hence higher crude oil prices drive fuel inflation as crude oil demand is inelastic. Diversity of participants like producers, government, extreme socio-political events and speculators drive crude oil market price.

The results demonstrate the overall significance of the crosscorrelation based on the analysis of multifractality. We found that the global Hurst coefficient varies with the $\mathrm{q}$ and there is multifractality evidenced through the multifractal spectrum also. We get the crosscorrelation exponent 0.48 . We found that there exists a power-law cross-correlation between the Gold and Crude Oil time series and the multifractal features are significant. The analysis throws light on the structure of crude oil and Gold market as well as its link to macroeconomic conditions and socio-political extreme events.

In this paper, we used multifractal detrended cross-correlation analysis to investigate the cross-correlation properties between Gold and WTI Crude Oil. We find that the cross-correlations display the characteristic of multifractality in the short term. Moreover, the crosscorrelations of small fluctuations are persistent, and those of large fluctuations are anti-persistent in the short term, while the crosscorrelations of all kinds of fluctuations are persistent in the long term.

Finally, when $\mathrm{q}<0$, the cross-correlation exponents are smaller than their average exponents, but larger than the average exponents when $\mathrm{q}>0$.

From our analysis we found Gold price has long term correlation with crude oil price. That is the reason why gold is sold off during economic weakness as it's also used as a kind of proxy currency. Currencies tend to lose their purchasing power over years due to inflation and as time passes it can't buy the same amount of oil which could have bought years ago while gold could. The main idea behind the gold-crude oil cross correlation is the one which suggests that prices of crude oil partly account for inflation. Increases in oil price increases prices of gasoline which is derived from oil which drives transport of 
Citation: Burugupalli S (2015) Multifractal Detrended Cross-correlation Analysis of Gold and WTI Crude Oil Price Time Series. J Bus Fin Aff 3: 130. doi:10.4172/2167-0234.1000130

Page 5 of 5

goods costly hence the Good prices rises. As a final result inflation hence gold price tend to appreciate with inflation rising. So, an increase in the price of crude oil can eventually translate into higher gold price.

\section{References}

1. Mandelbrot BB (1982) The Fractal Geometry of Nature, W H Freeman, New York.

2. Hurst HE (1951) Long-term storage capacity of reservoirs, Transactions of the American Society of Civil Engineers 116: 770-808.

3. Hurst HE (1957) A suggested statistical model of some time series which occur in nature, Nature 180: 494.

4. Peng CK, Havlin S, Stanley HE, Goldberger AL (1995) Quantification of scaling exponents and crossover phenomena in non-stationary heartbeat time series, Chaos 5: 82-90.

5. Tabak BM, Cajueiro DO (2007) Are the crude oil markets becoming weakly efficient over time? A test for time-varying long-range dependence in prices and volatility, Energy Economics 29: 28-36.

6. Buldyrev SV, Goldberger AL, Havlin S, Mantegna RN, Matsa ME, et al. (1995) Lang-range correlation properties of coding and noncoding DNA sequences: GenBank analysis, Physical Review E 51.

7. Podobnik B, Stanley HE (2008) Detrended cross-correlation analysis: a new method for analyzing two nonstationary time series, Physical Review Letters 100: 084102

8. Wang YD, Liu L (2010) Is WTI crude oil market becoming weakly efficient ove time? New evidence from multiscale analysis based on detrended fluctuation analysis, Energy Economics 32: 987-992.

9. Manimaran P, Pal M (2012) Dynamics of Fluctuations in Foreign Exchange Rate Time Series through Wavelet Analysis, Journal of Wavelet Theory and Applications 6: 1-10

10. Bolgorian M, Gharli Z (2011) A Multifractal Detrended Fluctuation Analysis of Gold Price Fluctuations, Act A Physic A Polonica B 42: 1

11. Lo AW (1991) Long term memory in stock market price, Econometrica 59 1279-1313.

12. Alvarez RJ, Cisneros M, Soriano A (2002) Multifractal Hurst analysis of crude oil prices, Physica A 313: 651-670.

13. Barabasi AL, Vicsek T (1991) Multifractality of self-affine fractals, Phys Rev A 44: $2730-2733$

14. Bunde A, Havlin S (1995) Fractals in Science, Springer-Verlag, Heidelberg, Germany.
15. Mantegna RN, Stanley HE (2000) Introduction to Econophysics: Correlations and Complexity in Finance, Cambridge University Press, Cambridge, UK.

16. Zhou WX (2008) Multifractal detrended cross-correlation analysis for two nonstationary signals, Physical Review E 77: 066211.

17. Wang YD, Wei Y, Wu C (2010) Cross-correlations between Chinese A-share and B-share markets, Physica A 389: 5468-5478.

18. Kantelhardt JW, Zschiegner SA, Koscienlny BE, Havlin S (2002) Multifracta detrended fluctuation analysis of nonstationary time series, Physica A 316: 87-114.

19. Manimaran $P$, Panigrahi PK, Parikh JC (2006) Difference in nature of correlation between NASDAQ and BSE indices, Physica A 387: 5810-5817.

20. Ma F, Wei Y, Huang D (2013) Multifractal detrended cross-correlation analysis between the Chinese stock market and surrounding stock markets, Physica A 392: $1659-1670$.

21. He LY, Chen SP (2011) Multifractal Detrended Cross-Correlation Analysis of agricultural futures markets, Chaos, Solitons \& Fractals 44: 355-361.

22. Horvatic D, Stanley HE, Podobnik B (2011) Detrended cross-correlation analysis for non-stationary time series with periodic trends, EPL 94: 18007.

23. Xue C, Shang P, Jing W (2012) Multifractal Detrended Cross-Correlation Analysis of BVP model time series, Nonlinear Dyn, 69:263-273.

24. Wang F, Liao G, Zhou X, Shi W (2013) Multifractal detrended cross-correlation analysis for power Markets, Nonlinear Dyn 72: 353-363.

25. Li Z, Lub X (2012) Cross-correlations between agricultural commodity futures markets in the US and China, Physica A 391: 3930-3941.

26. Cao G, Xuc L, Caoa J (2012) Multifractal detrended cross-correlations between the Chinese exchange market and stock market, Physica A 391: 4855-4866.

27. He LY, Fan Y, Wei YM (2009) Impact of speculator's expectations of returns and time scales of investment on crude oil price behaviors, Energy Economics 31: $77-84$.

28. Bernabe A Martinaa E, Alvarez-Ramirez J, Ibarra-Valdez C, (2004) A multimodel approach for describing crude oil price dynamics, Physica A 338: 567584.

29. Shao YH (2012) Comparing the performance of FA, DFA and DMA using different synthetic long-range correlated time series, SCIENTIFIC REPORTS 2: 835.

30. Caraiani P (2012) Evidence of Multifractality from Emerging European Stock Markets, PLoS ONE 7: 7 e40693. 